\title{
THE UNIVERSALITY OF THE VARIETY OF QUASIGROUPS
}

\author{
DON PIGOZZI \\ (Received 10 October 1974; revised 10 February 1975)
}

\begin{abstract}
The variety of quasigroups is universal for varieties of algebras of the most general kind in the sense that each such variety can be interpreted in a natural way in a suitably chosen subvariety of quasigroups. More precisely, for any algebra $\left\langle A, f_{0}, f_{1}, f_{2}, \cdots\right\rangle$ where $f_{0}, f_{1}, f_{2}, \cdots$ is an arbitrary finite or infinite sequence of operations of finite rank, there exists a quasigroup $\langle B, \cdot\rangle$ and polynomial operations $F_{0}, F_{1}, F_{2}, \cdots$ over $\langle B, \cdot\rangle$ such that $\left\langle A, f_{0}, f_{1}, \cdots\right\rangle$ is a subalgebra of $\left\langle B, F_{0}, F_{1}, \cdots\right\rangle$ satisfying exactly the same identities. Moreover, if there are only finitely many $f_{0}, f_{1}, \cdots$, then $\langle B, \cdot\rangle$ can be taken so that its identities are recursive in those of $\left\langle A, f_{0}, f_{1}, \cdots\right\rangle$. If $\left\langle A, f_{0}, f_{1}, \cdots\right\rangle$ is a free algebra with an infinite number of free generators, then $B$ can also be taken to coincide with $A$. This universal property of quasigroups has a number of consequences for their equational metatheory.
\end{abstract}

\section{Introduction}

A quasigroup is a groupoid in which each of the equations $a \cdot x=b$ and $y \cdot a=b$ has a unique solution for every pair of elements $a$ and $b$. Quasigroups have also been called "non-associative groups" because groups can be characterized exactly as those quasigroups whose multiplication is associative. In view of this it seems reasonable to expect that an attempt to develop a general theory of quasigroups along the lines of the theory of groups would prove profitable, and to a certain extent this has been borne out. (More success in this direction however has been obtained with the theory of loops.) On the other hand, there exists considerable evidence supporting the somewhat surprising conclusion that the general theory of quasigroups might have more in common with the theory of arbitrary groupoids than it has with that of groups. In this paper we shall attempt to bring this conclusion into sharper focus by proving a result that in a certain sense establishes the whole of the equational metatheory of algebraic structures of the most general kind as a part of the equational metatheory of quasigroups.

The research reported on here was supported in part by the National Science Foundation. 
The source of the parallelism between the theories of quasigroups and arbitrary algebras seems to lie mainly in the strong embedding results that have been obtained for quasigroups. For example, it is well known that every cancellation groupoid can be embedded in a quasigroup while the class of cancellation semigroups embeddable in groups is quite restricted; cf. Mal'cev (1939). Evans (1951) has shown that every partial loop can be embedded in a loop and uses this result to show that the general word problem for loops is solvable. In the same way it can be shown that every cancellation partial groupoid can be embedded in a quasigroup; thus the word problem for quasigroups is also solvable. This again is in marked contrast to the situation for groups. Evans (1971) formulates another criterion under which a partial groupoid on an infinite universe can be embedded in a loop with the same universe. Using this result he constructs a number of loops satisfying special identities, and then uses them to obtain various results about the lattice of loop varieties. In Bol'bot (1972) a closely related embedding lemma, although not explicitly formulated, is used to obtain other results on the structure of the lattice of quasigroup varieties. Finally, in Mal'cev (1966) a third version of the embedding lemma is used to prove that there exists a finitely based variety of quasigroups, in fact, commutative loops, whose set of identities is not recursive. The corresponding problem is still open in the case of groups.

The main result of this paper also assumes the form of an embedding result - one that in a restricted sense comprehends most of the known results of this kind for quasigroups. Loosely speaking it says that, if the notion of embedding one algebraic structure in another is generalized in a certain natural way, then the variety of quasigroups is universal with regard to embedding all other varieties in the following sense: every algebraic structure $\mathfrak{A}$ of arbitrary countable similarity type can be embedded in a quasigroup $\mathcal{B}$ in a way that preserves all the identities of $\mathscr{H}$. More precisely, for any algebra $\left\langle A, f_{0}, f_{1}, f_{2}, \cdots\right\rangle$ where $f_{0}, f_{1}, f_{2}, \cdots$ is an arbitrary finite or infinite sequence of operations of finite rank, there exists a quasigroup $\langle B, \cdot\rangle$ and polynomial operations $F_{0}, F_{1}, F_{2}, \cdots$ over $\langle B, \cdot\rangle$ such that $\left\langle A, f_{0}, f_{1}, \cdots\right\rangle$ is a subalgebra of $\left\langle B, F_{0}, F_{1}, \cdots\right\rangle$ satisfying exactly the same identities. Moreover, if there are only finitely many of the $f_{\kappa}$, then $\langle B, \cdot\rangle$ can be taken so that its identities are recursive in those of $\left\langle A, f_{0}, f_{1}, \cdots\right\rangle$; the quasigroup $\langle B, \cdot\rangle$ can also be chosen so that it fails to satisfy any finite number of identities specified beforehand, so long as they are not quasigroup identities. Finally, if $\left\langle A, f_{0}, f_{1}, \cdots\right\rangle$ is a free algebra with an infinite number of free generators, then $B$ can be taken to coincide with $A$.

This result has a number of consequences for the equational methatheory of quasigroups that provide simpler proofs and often considerable generalizations of many of the known results in this area. Several of these are presented in Section 3. 
The variety of quasigroups is just one of many known examples of varieties with the universal embedding property described above. Other examples are the varieties of commutative groupoids and of bisemigroups, that is, algebras with two independent associative operations. Although loops fail to form a universal variety, in Section 4 we discuss a partial result that has significant consequences for their metatheory. A general theory of universal varieties is developed in Pigozzi (a), (b). In particular, the first paper includes a test for universality that has wide applicability; although, unfortunately, it does not apply to quasigroups. Many of the results for quasigroups we shall present here are special cases of results of the general theory that are proved in detail in Pigozzi (b); in the sequel this paper shall be referred to by the mnemonic $\mathcal{U} \mathscr{E} \mathscr{T}$.

Let $\Gamma$ be a fixed but arbitrary set of defining identities for the variety of all quasigroups. In another paper, Pigozzi (b), we give a proof based on the results of the present paper that there exists no recursive algorithm for deciding whether or not a given finite set of identities, when taken together with $\Gamma$, defines a Schreier variety of quasigroups; a similar result is obtained for the amalgamation property. These results seem to be interesting in light of the fact that, in the case of groups, it is quite possible that algorithms exist for both these properties; cf. Neumann and Wiegold (1964) and Neumann (1967), Problem 6 and the following remark. We refer to the introduction of Pigozzi (b) for a more complete discussion of this problem.

\section{Preliminaries}

Our discussion shall be carried on within the context of the general theory of varieties as that theory is presented in Tarski (1968) and, in somewhat more detail, in $\mathscr{U} \mathscr{E} \mathscr{T}$. We shall explain all notation and terminology unless we are pretty sure it is commonly understood. But the discussion will be minimal and we refer the reader to the above mentioned papers for a more detailed exposition.

We shall be dealing with algebraic structures of the most general kind; in particular, algebras of arbitrary (similarity) type. In constructing the equational languages of all these various types we shall assume that the operation symbols are taken from a fixed universe of symbols and that a fixed positive rank has been assigned to each symbol in advance. Notice that we are excluding from consideration operation symbols of rank 0 , i.e., constant symbols, and hence by extension algebras which contain distinguished elements. Constants are treated in Tarski (1968) and $\mathscr{U} \mathscr{E} \mathscr{T}$, but not having to consider them here permits considerable technical simplification and their exclusion causes no real loss of generality; in this connection see the remarks immediately preceding Theorem 2.1 . 
It proves convenient to identify each similarity type with the set of operation symbols used in constructing the language of the type. Thus, if $I$ is an arbitrary type, the set of terms of type $I$ is the set of all strings of symbols one can form by starting with an infinite set $\boldsymbol{v}_{0}, \boldsymbol{v}_{1}, \boldsymbol{v}_{2}, \cdots$ of variable symbols and applying the operation symbols of $I$ over and over in a manner consistent with their ranks. The set of all such terms is denoted by $T e_{r}$. We use the prefix notation of Jan Kukasiewicz which allows us to avoid parentheses. For example, if $I=\{P, Q\}$ where $P$ and $Q$ are of rank 3 and 2 , respectively, then typical elements of $T e_{1}$ would be $v_{2}, Q v_{1} v_{2}, Q Q P v_{3} Q v_{0} v_{0} v_{0} Q v_{0} v_{0} v_{0}$; for brevity the last term can be written $Q^{2} P_{v_{3}}\left(Q v_{0}^{3}\right)^{2}$.

The left-most operation symbol of a non-variable term is called its principal operation symbol. If $\tau=Q \sigma_{0} \cdots \sigma_{\kappa-1}$, where $\kappa$ is the rank of $Q$, then $\sigma_{0}, \cdots, \sigma_{\kappa-1}$ are referred to as the principal subterms of $\tau$; if $Q$ is binary so that $\tau=Q \sigma_{0} \sigma_{1}$, then we write $\tau_{L}$ and $\tau_{R}$ for $\sigma_{0}$ and $\sigma_{1}$, respectively. We shall write $\sigma \leqslant \tau$ to indicate that $\sigma$ is a subterm of $\tau ; \sigma<\tau$ means $\sigma \leqslant \tau$ but $\sigma \neq \tau$. The length of a term, in symbols $|\tau|$, is just the total number of occurrences of operation and variable symbols. The set of all variable symbols is denoted by Va. Thus

$$
V a=\left\{\boldsymbol{v}_{0}, \boldsymbol{v}_{1}, \boldsymbol{v}_{2}, \cdots\right\} \text {. }
$$

We also write $x, y$, and $z$ for $v_{0}, v_{1}$, and $\boldsymbol{v}_{2}$, respectively. For any term $\tau, V a \tau$ denotes the set of all variable symbols that have at least one occurrence in $\tau$.

To indicate that a function $f$ is a mapping from a set $A$ into a set $B$ we shall write either $f: A \rightarrow B$ or $f \in{ }^{A} B$; if $A=B, f$ is called a transformation of $A$. Associated with each transformation $f$ of the set $T e_{I}$ of terms is another transformation $f^{*}$ of $T e_{I}$ defined by the conditions that $f^{*} v=v$ if $v \in V a$ and

$$
f^{*} Q \sigma_{0} \cdots \sigma_{\kappa-1}=Q f \sigma_{0} \cdots f \sigma_{\kappa-1}
$$

for all $Q \in I$ and $\sigma_{0}, \cdots, \sigma_{\kappa-1} \in T e_{1}$. (In this situation it is understood that $\kappa$ is the rank of $Q$.)

The substitution operators are important transformations of terms; there is one such operator associated with each assignment. By an assignment we mean any function from a subset of $V a$ into $T e_{1}$. For any assignment $\phi$ and term $\tau$ we use $s u_{\phi} \tau$ to denote the result of simultaneously replacing each occurrence in $\tau$ of a variable $v$ in the domain of $\phi$ by $\phi v ; s u_{\phi} \tau$ is called a substitution instance of $\tau$. If the domain of $\phi$ is the single variable $v$ and $\phi v=\sigma$, we write $s u_{\sigma}^{v} \tau$ in place of $s u_{\phi} \tau$. Also, if $\tau$ is any term and $\sigma_{0}, \cdots, \sigma_{\kappa-1}$ any sequence of terms, we shall write $\tau\left(\sigma_{0}, \cdots, \sigma_{\kappa-1}\right)$ for the particular substitution instance $s u_{\phi} \tau$ of $\tau$ where the assignment $\phi$ is given by $\phi v_{\lambda}=\sigma_{\lambda}$ for $\lambda<\kappa$ and $\phi \dot{v}_{\mu}=v_{\mu}$ for all $\mu \geqq \kappa$.

The equality symbol in all of our languages shall be $=$, and an equation of type $I$ is any formula of the form $\tau=\sigma$ where $\tau, \sigma \in T e_{r}$. The set of all equations of type $I$ is denoted by $E q_{r}$. 
We choose a special binary operation symbol $\boldsymbol{M}$ and reserve it exclusively to denote the multiplication operation of quasigroups. It is well known that the class of quasigroups forms a variety only when the two division operations are included along with multiplication as fundamental operations. Thus we also select two binary operation symbols $D_{L}$ and $D_{R}$ to denote the division operations and fix them throughout the discussion. We shall denote the similarity type of quasigroups by $\boldsymbol{T}$; thus

$$
T=\left\{M, D_{L}, D_{R}\right\} .
$$

Formally then, by a quasigroup we mean any algebra of type $T$ in which the following four equations are identically satisfied
(Ia) $M x D_{L} x y=y$
(Ib) $M D_{R} y x x=y$
(IIa) $D_{L} x M x y=y$
(IIb) $D_{R} M y x x=y$.

Each of the following two equations is easily seen to hold identically in every quasigroup
(IIIa)
$D_{R} x D_{L} y x=y$
(IIIb) $D_{L} D_{R} x y x=y$.

Let $\mathscr{K}$ be any class of algebras of the same similarity type $I$. By the theory of $\mathscr{K}$, in symbols $\Theta \mathscr{K}$, we mean the set of all equations which hold identically in every member of $\mathscr{K}$. If $\mathscr{K}=\{\mathscr{R}\}$ we write $\Theta \mathfrak{A}$ in place of $\Theta \mathscr{K}$. The theory of the class of quasigroups shall be denoted by $\boldsymbol{Q G}$. We often write $\sigma \equiv_{\theta} \tau$ instead of $(\sigma=\tau) \in \Theta$. If $\Gamma \subseteq E q_{I}$, then the theory generated by $\Gamma$, in symbols $\Theta_{I}[\Gamma]$, is the theory of the class of models of $\Gamma$; the set $\Gamma$ is called a base of $\Theta_{I}[\Gamma]$. For brevity we write $\sigma \equiv_{\Gamma} \tau$ in place of $\sigma \equiv_{\boldsymbol{\theta}[\Gamma]} \tau$. Thus $\boldsymbol{Q G}=\boldsymbol{\Theta}_{r}[\Gamma]$ where $\Gamma$ is the set of four equations (Ia), (Ib), (IIa), (IIb).

There is a well known purely syntactical characterization of $\boldsymbol{\theta}_{I}[\Gamma]$ in terms of $\Gamma$ that is due to Garrett Birkhoff. This characterization has many forms; the one we give here is particularly useful for our purposes. For every $\Gamma \subseteq T e_{\text {, we }}$ define a binary relation $\hat{=}_{\Gamma}$ on $T e_{r}$ in the following way: for all $\sigma, \tau \in T e_{r}$ we have $\sigma \hat{=}_{\Gamma} \tau$ iff there exist a $\pi \in T e_{I}$, a $v \in V a$, a $\phi \in{ }^{V a} T e_{I}$, and a pair of terms $\xi, \eta \in T e_{1}$ such that $(\xi=\eta) \in \Gamma$ or $(\eta=\xi) \in \Gamma$ and

$$
\sigma=s u_{\zeta}^{\mathrm{v}} \pi \quad \text { and } \quad \tau=s u_{\chi}^{\mathrm{v}} \pi \text {. }
$$

where $\zeta=s u_{\phi} \xi$ and $\chi=s u_{\phi} \eta$.

Then $\Theta_{I}[\Gamma]$ can be characterized as the transitive closure of the relation $\hat{=}_{\Gamma}$, i.e., $\Theta_{I}[\Gamma]$ is the set of all equations $\sigma=\tau$ such that there exists a sequence of terms $\xi_{i 1}, \cdots, \xi_{\kappa}$ such that $\xi_{0}=\sigma, \xi_{\kappa}=\tau$, and $\xi_{\lambda} \hat{=} \mathrm{r} \xi_{\lambda+1}$ for all $\lambda<\kappa$.

A normal-form function for an arbitrary theory $\Theta$ of type $I$ is a transformation $f$ of $T e_{I}$ such that (i) $f_{\tau} \equiv_{\Theta} \tau$ for every $\tau \in T e_{I}$; (ii) $\tau \equiv_{\Theta} \sigma$ implies $f \tau=f \sigma$ for all $\tau, \sigma \in T e_{I}$; (iii) $\sigma \leqslant f \tau$ implies $f \sigma=\sigma$ for all $\sigma, \tau \in T e_{I}$. A normal-form 
function $f$ for a theory $\Theta$ is said to be variable-minimizing if, for each $\tau \in T e_{1}$, $V a f \tau \subseteq V a \tau \cup\left\{v_{0}\right\}$, and $f \tau \dot{\epsilon}\left\{\tau, v_{0}\right\}$ if $\tau \in V a$. It is proved in $\mathscr{U E F}$, Theorem 2.5. that every theory $\Theta$ has a variable-minimizing normal-form function. If the type of $I$ is finite, $f$ can be taken to be recursive in $\Theta$; notice that in this case $f$ and $\Theta$ must have the same Turing degree of unsolvability.

Let $I$ and $J$ be arbitrary types. By a definition of $I$ in $J$ we shall mean any function $\rho: I \rightarrow T e_{J}$ such that, for each $Q \in I, V a \rho Q \subseteq\left\{v_{0}, \cdots, v_{\kappa-1}\right\}$ where $\kappa$ is the rank of $Q$. Let $\rho$ be a definition of $I$ in $J$ and let $\mathscr{A}=\left\langle A, R^{(\mathfrak{R})}\right\rangle_{R \in J}$ be an arbitrary algebra of type $J$ where $A$ is the universe of $\mathfrak{A}$ and, for each $R \in J$, $R^{(\mathfrak{a})}$ is the fundamental operation of $\mathfrak{A}$ corresponding to $R$. By the $\rho$-transform of $\mathfrak{A}$ we shall mean the algebra

$$
\mathfrak{I}_{\mathfrak{r}_{\rho}} \mathfrak{A}=\left\langle A, p l_{\mathfrak{r}}(\rho Q)\right\rangle_{O \in I}
$$

where $p l_{\mathfrak{l}}(\rho Q)$ is the polynomial operation over $\mathfrak{A}$ of the same rank as $Q$ that is defined in the natural way by the term $\rho Q$. Clearly $\mathfrak{I}_{\rho} \mathfrak{A}$ is of type $I$. Algebras are represented by capital German letters and their universes by the corresponding Roman letters.

DEFINITION 1.1. Let $J$ be any type and $\mathscr{K}$ a variety of type $J . \mathscr{K}$ is called unioersal if, for each countable type $I$, a definition $\rho$ of $I$ in $J$ can be found such that, for each algebra $\mathfrak{A}$ of type $I$, there exists a $\mathfrak{B} \in \mathscr{K}$ satisfying the following conditions:

(i) $\mathfrak{A}$ is a subalgebra of $\mathfrak{I r}_{\rho} \mathfrak{B}$;

(ii) $\Theta \mathfrak{U}=\Theta \mathfrak{T r}_{p} \mathfrak{B}$; i.e., $\mathfrak{A}$ and $\mathfrak{I}_{p} \mathfrak{B}$ satisfy exactly the same identities.

Assume now that $I$ is finite. If $\mathfrak{B}$ can always be taken so that, in addition to (i) and (ii),

(iii) $\Theta \mathcal{B}$ is recursive in $\Theta \mathscr{K}$ and $\Theta \mathfrak{U}$ together whenever $\mathfrak{A}$ is a free algebra over some variety, then $\mathscr{K}$ is said to be effectively universal.

A theory is universal, or effectively universal, if the variety of all its models has the property. In the sequel we shall pass back and forth between varieties and theories without hesitation, using whichever concept is the most convenient to deal with in each particular context.

If $I$ and $J$ are disjoint types and $\rho$ is a definition of $I$ in $J$, then for any pair of theories $\Theta$ and $\Phi$ of types $I$ and $J$, respectively, we define the $\rho$-coupling $\Theta \mathscr{C}_{\rho} \Phi$ of $\Theta$ and $\Phi$ by the equality

$$
\Theta \mathscr{C}_{\rho} \dot{\Phi}=\boldsymbol{\Theta}_{I \cup J}\left[\Theta \cup \Phi \cup\left\{Q v_{0} \cdots v_{\kappa-1}=\rho Q: Q \in I\right\}\right] .
$$

Observe that an arbitrary algebra $\mathfrak{A}=\left\langle A, Q^{(\mathfrak{a})}\right\rangle_{Q \in I \cup J}$ of type $I \cup J$ is a model of $\Theta \mathscr{C}_{p} \Phi$ iff (i) $\left\langle A, Q^{(A)}\right\rangle_{Q \in I}$. is a model of $\Theta$; (ii) $\left\langle A, Q^{(\mathfrak{a})}\right\rangle_{Q \in J}$ is a model of $\Phi$; and (iii) $\left\langle A, Q^{(2)}\right\rangle_{Q \in I}=\mathfrak{T}_{p}\left\langle A, Q^{(\mathfrak{A})}\right\rangle_{Q \in J}$. 
Definition 1.2. A definition $\rho$ of $I$ in $J$ is said to be non-overlapping if the following three conditions hold for every $Q \in I$ where $\kappa$ is the rank of $Q$.

(i) $\operatorname{Va} \rho \mathcal{Q}=\left\{v_{0} ; \cdots, v_{\kappa-1}\right\}$;

(ii) $\rho Q\left(\xi_{0}, \cdots, \xi_{\kappa-1}\right) \neq \sigma\left(\eta_{0}, \cdots, \eta_{\lambda-1}\right)$ for every non-variable $\sigma \leqslant \rho P$ and all $\xi_{0}, \cdots, \xi_{\kappa-1}, \eta_{0}, \cdots, \eta_{\lambda-1} \in T e_{I \cup J}$ where $P$ is any operation symbol of I distinct from $Q$ and $\lambda$ is its rank;

(iii) $\rho Q\left(\xi_{0}, \cdots, \xi_{\kappa-1}\right) \neq \sigma\left(\eta_{0}, \cdots, \eta_{\kappa-1}\right)$ for every non-variable $\sigma<\rho Q$ and all $\xi_{0}, \cdots, \xi_{\kappa-1}, \eta_{0}, \cdots, \eta_{\kappa-1} \in T e_{\text {IUJ }}$.

It is shown in McNulty (to appear), Theorem $2.9(\mathrm{v})$ (see also $\mathscr{U} \mathscr{E} \mathscr{T}$, Lemma 4.22 ) that, if $J$ contains at least one operation symbol of rank $\geqq 2$, then for each countable type $I$ there exists a non-overlapping definition $\rho$ of $I$ in $J$. For example, if $I=\{P, Q\}$ where $P$ and $Q$ are binary operation symbols, and if we take

$$
\rho P=M^{3} x^{3} M y M y^{2}, \quad \rho Q=M^{2} x M x^{2} M^{2} y^{3},
$$

then it is easy to check that $\rho$ is a non-overlapping definition of $I$ in $\{\boldsymbol{M}\}$.

For the purposes of the following definition, two types $I$ and $I^{\prime}$ are said to be isomorphic if there exists a one-one rank-preserving correspondence between them.

Definition 1.3. A non-overlapping definition $\rho$ of $I$ in $T$ is said to be special if all the following conditions are satisfied.

(i) Let $I^{\prime}=\left\{Q^{\prime}: Q \in I\right\}$ be a type isomorphic to I but disjoint from it, and let $\pi$ be the definition of $I \cup I^{\prime}$ in $T$ such that, for each $Q \in I, \pi Q=\rho Q_{2}$ and $\pi Q^{\prime}=\rho Q_{R}$ where $\rho Q_{L}$ and $\rho Q_{R}$ are the left-and right-hand principal subterms of $\rho Q$, respectively. Then $\pi$ is also non-overlapping.

Let $Q$ be any operation symbol in $I$ and let $\kappa$ be the rank of $Q$.

(ii) $M$ is the principal operation symbol of each of the terms $\rho Q, \rho Q_{L}$, and $\rho Q_{R}$.

(iii) $\rho Q$ contains no subterm of the form $D_{L} v \xi$ or $D_{R} \xi v$ with $v \in V a$.

(iv) For every non-variable $\sigma<\rho Q$ we have

$$
\sigma\left(\xi_{0}, \cdots, \xi_{\kappa-1}\right) \neq \gamma(\eta, \zeta)
$$

for all $\xi_{0}, \cdots, \xi_{\kappa-1}, \eta, \zeta \in T e_{I \cup T}$ and for every $\gamma$ which occurs as the left-hand side of one of the equations (Ia)-(IIIb).

Observe that, for every special non-overlapping definition $\rho$, conditions 1.2(i) and 1.3(i) together imply

$$
\operatorname{Va} \rho Q_{L}=\operatorname{Va} \rho Q_{R}=\operatorname{Va} \rho Q=\left\{v_{0}, \cdots, v_{k-1}\right\}
$$

for each $Q \in I$. 
The particular example of a non-overlapping definition $\rho$ considered in the remarks preceding 1.3 is not special. But special non-overlapping definitions are easy to construct as we see from the proof of the following lemma.

LEMMA 1.4. For every countable type I there exists a special non-overlapping definition of I in $\boldsymbol{T}$; moreover it can be taken so that its range is included in $T e_{\{M\}}$.

Proof. Let $I^{\prime}=\left\{Q^{\prime}: Q \in I\right\}$ be a type isomorphic to $I$ but disjoint from it. By $\mathscr{U} \mathscr{E} \mathscr{T}$, Lemma 4.22, there exists a non-overlapping definition $\pi$ of $I \cup I^{\prime}$ in $\{M\}$. Moreover, from the proof of 4.22 given in $\mathscr{U} \mathscr{E} \mathscr{T}$ it is immediately seen that, for every $Q \in I \cup I^{\prime}, \pi Q$ has the following property.

(1) If $\sigma \leqslant \rho Q$ and $\sigma \notin V a$, but at least one of the two principal subterms of $\sigma$ is a variable, then $\sigma$ must be in one of the three forms $\boldsymbol{M} v^{2}, \boldsymbol{M}^{2} v^{3}$, or $\boldsymbol{M} v \boldsymbol{M} v^{2}$.

For each $Q \in I$ take

$$
\rho Q=M \pi Q \pi Q^{\prime} .
$$

Conditions 1.2(i) and 1.3(i)-(iii) are immediate consequences of the fact $\pi$ is a non-overlapping definition of $I \cup I^{\prime}$ in $\{M\}$. Consider any non-variable $\sigma<\rho Q$ where $Q \in I$ and suppose

$$
\sigma\left(\xi_{0}, \cdots, \xi_{\kappa-1}\right)=\gamma(\eta, \zeta)
$$

where $\gamma=y$ is one of the equations (Ia)-(IIIb). Because the principal operation symbol of $\sigma$ is necessarily $M, \gamma=y$ must be (Ia) or (Ib). If $\gamma=y$ is (Ia), then, as $\sigma$ contains no occurrence of $D_{L}$, the right-hand principal subterm of $\sigma$ must be a variable. From (1) and (3) we could then conclude that for some $\lambda<\kappa$,

$$
\lambda<\kappa ; M \eta D_{L} \eta \zeta \in\left\{M \xi_{\lambda}^{2}, M^{2} \xi_{\lambda}^{3}, M \xi_{\lambda} M \xi^{2}\right\} .
$$

This is clearly impossible, and a similar result is obtained if $\gamma=\boldsymbol{y}$ happens to be (Ib) instead of (Ia). Thus 1.3(iv) holds.

To verify 1.2 (ii) consider any two distinct $Q, P \in I$ of ranks $\kappa$ and $\lambda$, respectively, and let $\sigma$ be any non-variable subterm of $\rho P$. Assume that for some $\xi_{0}, \cdots, \xi_{\kappa-1}, \eta_{0}, \cdots, \eta_{\lambda-1}$ we have

$$
\rho Q\left(\xi_{0}, \cdots, \xi_{\kappa-1}\right)=\sigma\left(\eta_{0}, \cdots, \eta_{\lambda-1}\right) .
$$

If $\sigma=\rho P$, then (2) and (4) imply $\pi Q\left(\xi_{0}, \cdots, \xi_{\kappa-1}\right)=\pi P\left(\eta_{0}, \cdots, \eta_{\lambda-1}\right)$ which contradicts the fact that $\pi$ is non-overlapping. Thus $\sigma \leqslant \pi P$ or $\sigma \leqslant \pi P^{\prime}$. But in this case (4) together with the non-overlapping property of $\pi$ implies that $\sigma$ is of the form $M v u$ with $v, u \in V a$. Applying (1) with $P$ in place of $Q$ we get $v=u$, and thus finally, from (2) and (4), $\pi Q\left(\xi_{0}, \cdots, \xi_{\kappa-1}\right)=\pi Q^{\prime}\left(\xi_{0}, \cdots, \xi_{\kappa-1}\right)$. This again

\footnotetext{
2 This result was first announced in Pigozzi (1973).
} 
contradicts the fact that $\pi$ is non-overlapping, so we conclude that 1.2(ii) holds. Condition 1.2(iii) is verified in the same way. Thus the lemma is proved.

The special non-overlapping definitions constructed in the preceding proof are probably the simplest available. Others can be found however that are more complicated but have additional properties that prove to be useful for various purposes. For example the special non-overlapping definitions obtained in the following lemma are used in Pigozzi (b) to prove that there exists no recursive algorithm for deciding whether or not a given finite set of equations to type $\boldsymbol{T}$ defines the variety of groups when (Ia)-(IIb) are adjoined.

LEMMA 1.5. Let $I$ be any countable type and let $P$ be a binary operation symbol not contained in I. Let $\mathbf{G R}$ be the theory of groups of type $\boldsymbol{T}$, i.e., the theory generated by $(I a)-(I l b)$ together with the associative law $\boldsymbol{M}^{2} \boldsymbol{x y z}=\mathbf{M x} \boldsymbol{M y z}$.

There exists a special non-overlapping definition $\rho$ of $I \cup\{P\}$ in $T$ such that $\rho P \equiv{ }_{\text {GR }} \boldsymbol{x}$.

Proof. Let $I^{\prime}$ and $\pi$ be as in the proof of 1.4 and assume $P \notin I^{\prime}$. For each $Q \in I$ take $\rho Q$ as in (2) and take

$$
\rho P=M^{2} D_{R}^{2} x M x^{2} M^{2} y^{3} M y M y^{2} M B_{R} M x^{2} M^{2} y^{3} M y M y^{2} .
$$

The proof that all the conditions of 1.2 and 1.3 are satisfied is straightforward; we shall omit the details. Also it is easy to check that $\rho P \equiv_{G R} x$.

\section{Main Lemma}

The central lemma of this paper, from which all our results follow, has a purely combinatorial character. Among other things it says that, for each countable similarity type $I$, there exists a definition $\rho$ of $I$ in $\boldsymbol{T}$ (in fact in $\{\boldsymbol{M}\}$ ) such that, for every theory $\Theta$ of type $I$, the process of $\rho$-coupling $\Theta$ with $Q \boldsymbol{G}$ does not lead to any new identities of type $I$, i.e.,

$$
\left(\Theta \mathscr{C}_{\rho} Q G\right) \cap E q_{I}=\Theta
$$

We prove this result by constructing a normal-form function for $\Theta \mathscr{C}_{\rho} Q \boldsymbol{G}$ which when restricted to equations of type $I$ becomes a normal-form function for $\Theta$. But this normal-form function has additional properties which make it a powerful tool in studying the properties of the polynomial embedding of arbitrary algebras in quasigroups. The particular method of construction we use results from combining methods of Evans (1951) and the author $\mathscr{U} \mathscr{E} \mathscr{T}$.

In order to simplify our combinatorial arguments we shall assume, as indicated in the Preliminaries, that all operation symbols under consideration are of positive rank. This amounts to no real loss of generality since constant unary operations can in a natural way be interpreted as operations of rank 0 . 
More precisely, given an arbitrary theory $\Theta$ of type $I$, the constant symbols of $I$ (relative to $\Theta$ ) will be identified with those unary operation symbols $Q$ of $I$ such that $\Theta$ contains the equation $Q x=Q y$.

Theorem 2.1. Assume $I$ is any countable type disjoint from $\boldsymbol{T}$ and $\Theta$ any arbitrary consistent theory of type I. Assume also that $\rho$ is a special nonoverlapping definition of $I$ in $T$. Then there exists a transformation $m$ of $T e_{r \cup T}$ satisfying all of the following conditions.

(i) $m$ is a normal-form function for $\Theta \mathscr{C}_{p} Q G$.

(ii) $m$ restricted to $T e_{1}$ is a normal-form function for $\Theta$.

(iii) Consider any $\tau, \tau^{\prime} \in T e_{1}$ and assume that $V a \tau \cup V a \tau^{\prime} \subseteq\left\{v_{0}, \cdots, v_{\kappa-1}\right\}$. Let $\sigma_{0}, \cdots, \sigma_{\kappa-1}$ be a sequence without repetitions of terms in $T e_{r \cup T}$ such that, for each $\lambda<\kappa, m \sigma_{\lambda}=\sigma_{\lambda}$ and $\sigma_{\lambda}$ is either a variable, or has its principal operation symbol in $T$. Then

$$
m\left(\tau\left(\sigma_{0}, \cdots, \sigma_{\kappa-1}\right)\right)=m\left(\tau^{\prime}\left(\sigma_{0}, \cdots, \sigma_{\kappa-1}\right)\right) \quad \text { iff } \quad m \tau=m \tau^{\prime} .
$$

In addition, if $I$ is finite, then

(iv) $m$ is recursive in $\Theta$.

Before beginning the proof we introduce some useful notions of a rather technical nature. Consider an arbitrary term $\tau \in T e_{r \cup r}$ and a sequence $\sigma_{0}, \cdots, \sigma_{\kappa-1}$ of disjoint occurrences of subterms of $\tau$. We shall want to have available a canonical method for replacing the $\sigma_{\lambda}$ in $\tau$ by variables so as to obtain a new term $\tau^{\prime}$ with the property that $\tau$ can be retrieved from $\tau^{\prime}$ by substituting terms for variables in the usual way. The most convenient way to do this is to enlarge our language by adjoining a new variable for each of the old non-variable terms in $\mathrm{Te}_{\boldsymbol{I} \cup \mathrm{T}}$. Let $\mathrm{Va}^{+}$be the set of variables with these new variables adjoined, and let $\chi$ be a one-one recursive function from $\mathrm{Va}^{+}$onto $T e_{r \cup r}$ such that $\chi v=v$ for each $v \in V a ; \chi$ is assumed to be fixed throughout the whole discussion. Let $\mathrm{Te}_{1}^{+}$denote the set of all terms of type $I$ that possibly contain occurrences of new variables as well as old ones; let $\mathrm{Te}_{I}^{++}$be the set of a:l $\tau \in T e_{t}^{+}$such that for each $v \in V a^{+}$, if $v \leqslant \tau$, then either $\chi v \in V a$ (and hence $\boldsymbol{x} v=v$ ) or the principal operation symbol of $\chi v$ is contained in $T$. The following lemma is now immediate.

(1) Let $\tau \in T e_{I \cup T}$. Then there exists a unique $\sigma \in T e_{I}^{++}$such that $\tau=s u_{x} \sigma$. Let

$$
\Theta^{+}=\left\{\sigma\left(u_{0}, \cdots, u_{\kappa-1}\right)=\tau\left(u_{0}, \cdots, u_{\kappa-1}\right): \sigma=\tau \in \Theta \text { and } u_{0}, \cdots, u_{\kappa-1} \in V a^{+}\right\} .
$$

Clearly $\Theta^{+}$is a theory of type $I$ in the extended language such that $\Theta^{+} \cap E q_{1}=$ $\Theta$. Furthermore $\Theta^{+}$is obviously recursively isomorphic to $\Theta$. As was mentioned in the Preliminaries it is proved in $\mathscr{U} \mathscr{E} \mathscr{T}$, Theorem 2.5 , that there exists a 
variable-minimizing normal-form function for $\Theta^{+}$that is recursive in $\Theta^{+}$in case $I$ is finite. Let $f$ be one such function which we assume to be fixed throughout the discussion. Notice that, since $f$ is variable-minimizing, it becomes a normal-form function for $\Theta$ when restricted to $T e_{I}$. Finally, let $\bar{f}$ be the transformation of $T e_{I \cup T}$ whose value at each $\tau \in T e_{I \cup T}$ is given by

$$
\bar{f} \tau=s u_{x} f \sigma
$$

where $\sigma$ is the unique term in $T e_{I}^{++}$such that $\tau=s u_{x} \sigma$.

We are now ready to construct the transformation $m$ of $T e_{I \cup T}$ whose existence is asserted in the statement of 2.1. Let $\rho$ be any special nonoverlapping definition of $I$ in $T$. For each $Q \in I$ we shall write

$$
\rho Q=M \rho Q_{L} \rho Q_{R}
$$

Observe that, because $\rho$ is both special and non-overlapping, both $\rho Q_{L}$ and $\rho Q_{R}$ contain at least one occurrence of $v_{\lambda}$ for each $\lambda$ less than the rank of $Q$. The transformation $m$ is defined by recursion on the length of terms. If $\tau \in V a$, then we take $m \tau=\tau$. Assume $|\tau|>1$. The definition of $m \tau$ separates into six cases which we shall later show to be mutually exclusive.

CASE 1: $m^{*} \tau=\sigma(\xi, \eta)$ where $\sigma=y$ is one of the six quasigroup identities (Ia)-(IIIb) given in the Preliminaries. Take $m \tau=\eta$.

CASE 2: $m^{*} \tau=D_{L} \rho Q_{L}\left(\xi_{0}, \cdots, \xi_{\kappa-1}\right) \bar{f} Q \xi_{0} \cdots \xi_{\kappa-1}$ where $Q$ is some operation symbol of $I$ and $\kappa$ is the rank of $Q$. Take $m \tau=\rho Q_{R}\left(\xi_{0}, \cdots, \xi_{\kappa-1}\right)$.

CASE 3: $m^{*} \tau=D_{R}\left(\bar{f} Q \xi_{0} \cdots \xi_{\kappa-1}\right) \rho Q_{R}\left(\xi_{0}, \cdots, \xi_{\kappa-1}\right)$ where $Q$ is some operation symbol of $I$ and $\kappa$ is the rank of $Q$. Take $m \tau=\rho Q_{L}\left(\xi_{0}, \cdots, \xi_{\kappa-1}\right)$.

CASE 4: $m^{*} \tau=\rho Q\left(\xi_{0}, \cdots, \xi_{k-1}\right)$ where $Q$ is some operation symbol of $I$ and $\kappa$ is the rank of $Q$. Take $m \tau=\bar{f} Q \xi_{0} \cdots \xi_{\kappa-1}$.

CASE 5: $m^{*} \tau=Q \xi_{0} \cdots \xi_{\kappa-1}$ where $Q \in I$ and $\kappa$ is the rank of $Q$. Take $m \tau=\bar{f} m^{*} \tau$.

CASE 6: None of the five previous cases apply. Take $m \tau=m^{*} \tau$.

To show that $m$ is well defined we must first show that the six cases are mutually exclusive. Assume first of all that Case 1 applies so that $m^{*} \tau=\sigma(\eta, \zeta)$ where $\sigma=y$ is one of the six identities (Ia)-(IIIb).

Suppose Case 2 also applies so that

$$
m^{*} \tau=\boldsymbol{D}_{L} \rho Q_{L}\left(\xi_{0}, \cdots, \xi_{\kappa-1}\right) \bar{f} Q \xi_{0} \cdots \xi_{\kappa-1} .
$$

Then $\sigma=y$ must be either (IIa) or (IIIb). In the first case we would have $m^{*} \tau=\boldsymbol{D}_{L} \eta \boldsymbol{M} \eta \zeta$, and hence $\eta=\rho Q_{L}\left(\xi_{0}, \cdots, \xi_{\kappa-1}\right)$ and

$$
\bar{f} Q \xi_{0} \cdots \xi_{\kappa-1}=\boldsymbol{M} \eta \zeta=\boldsymbol{M} \rho Q_{L}\left(\xi_{0}, \cdots, \xi_{\kappa-1}\right) \zeta .
$$


Let $Q \xi_{0} \cdots \xi_{\kappa-i}=s u_{x} \gamma$ with $\gamma \in T e_{I}^{++}$. Then $\bar{f} Q \xi_{0} \cdots \xi_{\kappa-i}=s u_{x} f \gamma$. It follows that $f \gamma \in V a^{+}$since, by (3), the principal operation symbol of $\bar{f} Q \xi_{0} \cdots \xi_{\kappa-1}$ is $M$. Thus

$$
\bar{f} Q \xi_{0} \cdots \xi_{\kappa-1} \leqslant \xi_{\lambda} \text { for some } \lambda<\kappa .
$$

But $\xi_{\lambda} \leqslant \rho Q_{L}\left(\xi_{0}, \cdots, \xi_{\kappa-1}\right)$ since $\rho$ is special ; combining this with (3) and (4) we obtain a contradiction. Therefore, $\sigma=y$ must be (IIIb) and hence $m^{*} \tau=$ $D_{L} D_{R} \eta \zeta \eta$. Together with (2) this implies that $D_{R} \eta \zeta=\rho Q_{L}\left(\xi_{0}, \cdots, \xi_{\kappa-1}\right)$ which is impossible since $\rho Q_{L}$ has $M$ as its principal operation symbol by 1.3 (ii). Thus Cases 1 and 2 cannot simultaneously apply and by symmetry neither can Cases 1 and 3 .

It is also not possible for Cases 1 and 4 to apply simultaneously since Case 4 implies that $\boldsymbol{M}$ is the principal operation symbol of $m^{*} \tau$ and both its principal subterms, but this is obviously not possible in Case 1. Finally, again considering the principal operation symbol of $m^{*} \tau$ we see that Cases 1 and 5 are mutually exclusive. Similar considerations easily lead to the conclusion that each of the Cases 2, 3, and 4 exclude the remaining five cases.

To complete the argument that $m$ is well defined we must show that, for each of the six cases, when a particular case applies the value of $m \tau$ is uniquely determined. In Cases 5 and 6 this is obvious. In Case 1 it amounts to showing that if $\sigma$ and $\sigma^{\prime}$ are the left-hand sides of distinct equations among (Ia)-(IIIb), then $\sigma(\xi, \eta) \neq \sigma^{\prime}\left(\xi^{\prime}, \eta^{\prime}\right)$ for all $\xi, \eta, \xi^{\prime}, \eta^{\prime} \in T e_{r \cup r}$. But this non-equality is easily checked. For instance, considering the equations (Ia) and (Ib) we have $\sigma(\xi, \eta)=$ $\boldsymbol{M} \xi \boldsymbol{D}_{\mathrm{L}} \xi \boldsymbol{\eta}$ and $\sigma^{\prime}\left(\xi^{\prime}, \eta^{\prime}\right)=\boldsymbol{M} \boldsymbol{D}_{\mathrm{R}} \eta^{\prime} \xi^{\prime} \xi^{\prime}$; then $\sigma(\xi, \eta)=\sigma^{\prime}\left(\xi^{\prime}, \eta^{\prime}\right)$ implies the obvious contradiction $D_{L} D_{R} \eta^{\prime} \xi^{\prime} \eta=\xi^{\prime}$.

To show that $m \tau$ is uniquely defined if Case 2 applies it clearly suffices to show that, for each $Q \in I$ and all $\xi_{0}, \cdots, \xi_{\kappa-1} \in T e_{r \cup r}, \rho Q\left(\xi_{0}, \cdots, \xi_{\kappa-1}\right)$ and hence $\rho Q_{R}\left(\xi_{0}, \cdots, \xi_{\kappa-1}\right)$ are uniquely determined by $\rho Q_{L}\left(\xi_{0}, \cdots, \xi_{\kappa-1}\right)$. This result however follows immediately from the assumption that the definition $\pi$ defined in 1.3(i) is non-overlapping. By symmetry $m \tau$ is uniquely defined in the event Case 3 applies, and the non-overlapping property of $\rho$ also leads immediately to the conclusion that $m \tau$ is uniquely determined in the event of Case 4 . This completes the demonstration that $m$ is well defined. Also it is not difficult to see that, when $I$ is finite, $m$ is recursive in $f$, and hence also in $\Theta$ since $f$ is taken to be recursive in $\Theta$. This verifies condition 2.1(iv).

We now turn to the task of verifying the first three conditions of the conclusion of 2.1. This will be done in a sequence of lemmas, 2.2 through 2.9. In these lemmas $I, \Theta$, and $\rho$ are assumed to be as in the statement of $2.1, f$ is a variable-minimizing normal-form function for $\Theta^{+}$, recursive in $\Theta$ in case $I$ is finite, and $m$ is constructed from $f$ and $\rho$ by Cases $1-6$.

Lemma 2.2. Consider any $Q \in I$ and let $\kappa$ be the rank of $Q$. Then for any $\sigma<\rho Q$ we have 


$$
m\left(\sigma\left(\xi_{0}, \cdots, \xi_{\kappa-1}\right)\right)=\sigma\left(\xi_{0}, \cdots, \xi_{\kappa-1}\right)
$$

for all $\xi_{0}, \cdots, \xi_{\kappa-1} \in T e_{I \cup T}$ such that $m \xi_{\lambda}=\xi_{\lambda}$ for all $\lambda<\kappa$.

Proof. by induction on the length of $\sigma$. If $\sigma$ is a variable, the result is obvious. Assume

$$
\sigma=R \gamma \delta
$$

with $R \in T$. Let

$$
\tau=\sigma\left(\xi_{0}, \cdots, \xi_{\kappa-1}\right)=R \gamma\left(\xi_{0}, \cdots, \xi_{\kappa-1}\right) \delta\left(\xi_{0}, \cdots, \xi_{\kappa-1}\right) .
$$

By the induction hypothesis we have $m^{*} \tau=\tau$.

In the definition of $m \tau$ Case 1 cannot apply because of 1.3(iv). Suppose Case 2 applies so that

$$
\tau=D_{L} \rho P_{L}\left(\eta_{0}, \cdots, \eta_{\lambda-1}\right) \bar{f} P \eta_{0} \cdots \eta_{\lambda-1}
$$

for some $P \in I$. Then from (5) we get $\gamma\left(\xi_{0}, \cdots, \xi_{\kappa-1}\right)=\rho P_{L}\left(\eta_{0}, \cdots, \eta_{\lambda-1}\right)$. By 1.3(i) this is impossible unless $\gamma$ is a variable; but, by 1.3(iii), $\gamma$ cannot be a variable since $R=D_{L}$ by (5) and (6). Hence, Case 2 cannot apply, and by symmetry, neither can Case 3.

Case 5 is excluded because the principal operation symbol of $\sigma$ is in $T$, and Case 4 is excluded because $\rho$ is non-overlapping. Thus Case 6 applies and we have $m \tau=m^{*} \tau=\tau$ as was to be shown.

LEMMA 2.3. $\sigma \leqslant m \tau$ implies $m \sigma=\sigma$ for all $\sigma, \tau \in T e_{I} \cup \boldsymbol{T}$.

Proof. by induction on the length of $\tau$. The desired result clearly holds if $\tau \in V a$, and we assume that $\tau=P \pi_{0} \cdots \pi_{\kappa-1}$ for some $P \in I \cup T$. Then

$$
m^{*} \tau=P m \pi_{0} \cdots m \pi_{\kappa-1} \text {. }
$$

If Case 1 applies, then $\sigma \leqslant m \pi_{\lambda}$ for $\lambda \in\{0,1\}$, and hence $m \sigma=\sigma$ by the induction hypothesis. If Case 2 applies, then

$$
m^{*} \tau=D_{L} \rho Q_{L}\left(\xi_{0}, \cdots, \xi_{\kappa-1}\right) \bar{f} Q \xi_{0} \cdots \xi_{\kappa-1}
$$

and

$$
\sigma \leqslant \rho Q_{R}\left(\xi_{0}, \cdots, \xi_{\kappa-1}\right) .
$$

From (7), (8), and 1.3(i) (see the remark immediately following 1.3) we have $\xi_{\lambda} \leqslant m \pi_{0}$ for each $\lambda<\kappa$. Hence, by the induction hypothesis, $m \xi_{\lambda}=\xi_{\lambda}$ for every such $\lambda$. The desired conclusion $m \sigma=\sigma$ now follows immediately from (9) and 2.2. By symmetry we obtain the same result if Case 3 applies.

Suppose now that Case 5 applies so that $P$ in $(3)$ is contained in $I$. Then

$$
m \tau=\bar{f} m^{*} \tau=s u_{x} f \gamma
$$


where $\gamma$ is the unique term in $T e_{I}^{++}$such that $m^{*} \tau=s u_{x} \gamma$. By (7) we have that, for each $v \in V a \gamma$, there exists a $\lambda<\kappa$ such that $\chi v \leqslant m \pi_{\lambda}$. Hence by the induction hypothesis and the fact that $f$ is variable-minimizing we get

$$
m \chi v=\chi v \text { for all } v \in V a f \gamma .
$$

From (10) we have that $\sigma \leqslant s u_{x} f \gamma$; thus either $\sigma \leqslant \chi v$ for some $v \in V a f \gamma$, or $\sigma=s u_{x} \sigma^{\prime}$ for some $\sigma^{\prime} \leqslant f \gamma$. In the first case we have $m \sigma=\sigma$ by (11) and the induction hypothesis; in the second case the same result can be obtained, using (11), by a simple induction on the length of $\sigma^{\prime}$.

Finally, we assume that Case 4 holds. Then $m^{*} \tau=\rho Q\left(\xi_{0}, \cdots, \xi_{\kappa-1}\right)$. Thus, since $m \xi_{\lambda}=\xi_{\lambda}$ for all $\lambda<\kappa$ by (7) and the induction hypothesis, we can also apply Case 5 and get

$$
m \tau=m Q \xi_{0} \cdots \xi_{\kappa-1}=s u_{x} f \gamma
$$

where $Q \xi_{0} \cdots \xi_{\kappa-1}=s u_{x} \gamma$ with $\gamma \in T e_{I}^{++}$. The argument now proceeds as in the treatment of Case 5. This completes the proof of the lemma.

LEMMA 2.4. $m s u_{\phi} \tau=m s u_{m \circ \phi} \tau$ for every $\tau \in T e_{I \cup T}$ and every $\phi \in{ }^{\mathrm{va}} T e_{I \cup T}$.

Proof. by induction on the length of $\tau$. If $\tau \in V a$, then, by $2.3, m s u_{\phi} \tau=$ $m \phi \tau=m m \phi \tau=m s u_{m \circ \phi} \tau$. Let $\quad \tau=Q \pi_{0} \cdots \pi_{\kappa-1}$. Then $\quad m^{*} s u_{\phi} \tau=$ $Q m s u_{\phi} \pi_{0} \cdots m s u_{\phi} \pi_{\kappa-1}=Q m s u_{m \circ \phi} \pi_{0} \cdots m s u_{m \circ \phi} \pi_{\kappa-1}=m{ }^{*} s u_{m \circ \phi} \tau$. The equality $m s u_{\phi} \tau=m s u_{m \circ \phi} \tau$ now follows directly from the definition of $m$.

LeMma 2.5. $m\left(\rho Q\left(\xi_{0}, \cdots, \xi_{\kappa-1}\right)\right)=m Q \xi_{0} \cdots \xi_{\kappa-1}$ for all $Q \in I$ and $\xi_{0}, \cdots, \xi_{\kappa-1} \in T e_{I \cup T}$.

Proof. By 2.3 and 2.4 we assume without loss of generality that $m \xi_{\lambda}=\xi_{\lambda}$ for all $\lambda<\kappa$. Then by 2.2 we have

$$
m^{*}\left(\rho Q\left(\xi_{0}, \cdots, \xi_{\kappa-1}\right)\right)=\rho Q\left(\xi_{0}, \cdots, \xi_{\kappa-1}\right) .
$$

Thus by Cases 4 and 5 we have that

$$
m\left(\rho Q\left(\xi_{0}, \cdots, \xi_{\kappa-1}\right)\right)=\bar{f} Q \xi_{0} \cdots \xi_{\kappa-1}=m Q \xi_{0} \cdots \xi_{\kappa-1}
$$

LEMMA 2.6. $m s u_{\phi} \tau=\bar{f} s u_{m \circ \phi} \tau$ for all $\tau \in T e_{I}$ and $\phi \in E^{v a} T e_{I \cup T}$.

Proof. by induction on the length of $\tau$. Before starting the proof we show that

$$
\vec{f} m \sigma=m \sigma \text { for all } \sigma \in T e_{I \cup T}
$$

To see this observe that, if the principal operation symbol of $m \sigma$ is in $I$, then by Case 5 and 2.3 we have 


$$
m \sigma=m m \sigma=\bar{f} m^{*} m \sigma=\bar{f} m \sigma ;
$$

otherwise we get $\bar{f} m \sigma=m \sigma$ directly from the definition of $\bar{f}$.

If $\tau \in V a$, then, by (12), $m s u_{\phi} \tau=m \phi \tau=\bar{f} m \phi \tau=\bar{f} s u_{m \circ \phi} \tau$. Assume that $\tau$ has length 2 or more. Then the principal operation symbol of $s u_{\phi} \tau$ is in $I$, and thus Case 5 applies in the definition of $m s u_{\phi} \tau$. Hence by the induction hypothesis we have

$$
m s u_{\phi} \tau=\bar{f} m^{*} s u_{\phi} \tau=\overline{f f}^{*} s u_{m \circ \phi} \tau \text {. }
$$

Since $f$ is a variable-minimizing normal-form function, it is easy to check that $\bar{f}^{*} s u_{m \circ \phi} \tau=\bar{f} s u_{m \circ \phi} \tau$. This completes the proof of the lemma.

LEMma 2.7. For all $\xi, \eta, \zeta \in T e_{r \cup T}$ such that $m \xi=\xi, m \eta=\eta$, and $m \zeta=\zeta$ the following three conditions are equivalent:

(i) $m \boldsymbol{M} \xi \eta=\zeta$;

(ii) $m D_{L} \xi \zeta=\eta$;

(iii) $m D_{R} \zeta \eta=\xi$.

Proof. We shall only prove the equivalence of (i) and (ii); the equivalence of (i) and (iii) will follow by symmetry.

Assume that (i) holds and observe that $m^{*} \boldsymbol{M} \xi \boldsymbol{\eta}=\boldsymbol{M} \xi \boldsymbol{\eta}$ by hypothesis. In the evaluation of $m \boldsymbol{M} \xi \eta$ the cases that can apply are Case 1 with $\boldsymbol{\sigma}=\boldsymbol{y}$ either (Ia) or (Ib), and Cases 4 and 6. We consider each case separately.

Case 1-(Ia). Then $\boldsymbol{M} \xi \eta=M \xi D_{L} \xi \zeta$ so that $D_{L} \xi \zeta=\eta$, and thus (ii) holds since $m \eta=\eta$ by hypothesis.

Case 1-(Ib). Then $M \xi \eta=M D_{R} \zeta \eta \eta$ so that $\xi=D_{R} \zeta \eta$. Then $D_{L} \xi \zeta=$ $D_{L} D_{R} \zeta \eta \zeta$. Hence since

$$
m^{*} \boldsymbol{D}_{L} \xi \zeta=\boldsymbol{D}_{L} \xi \zeta
$$

holds by assumption, Case 1-(IIIb) applies in the evaluation of $m D_{L} \xi \zeta$, and thus we again get (ii).

Case 4. Then for some $Q \in I$ and $\xi_{0}, \cdots, \xi_{\kappa-1} \in T e_{I \cup T}$ we have

$$
\boldsymbol{M} \xi \eta=\rho Q\left(\xi_{0}, \cdots, \xi_{\kappa-1}\right)=\boldsymbol{M} \rho Q_{L}\left(\xi_{0}, \cdots, \xi_{\kappa-1}\right) \rho Q_{R}\left(\xi_{0}, \cdots, \xi_{\kappa-1}\right)
$$

and $\zeta=m \boldsymbol{M} \xi \eta=\bar{f} Q \xi_{0} \cdots \xi_{\kappa-1}$. Then

$$
\boldsymbol{D}_{L} \xi \dot{\zeta}=\boldsymbol{D}_{L} \rho Q_{L}\left(\xi_{0}, \cdots, \xi_{\kappa-1}\right) \bar{f} Q \xi_{0} \cdots \xi_{\kappa-1} .
$$

Thus, since (13) holds, Case 2 applies in the evaluation of $m D_{L} \xi \zeta$, and, by (14), $m D_{L} \xi \zeta=\rho Q_{R}\left(\xi_{0}, \cdots, \xi_{\kappa-1}\right)=\eta$, so (ii) holds again.

Case 6. $\zeta=m \boldsymbol{M} \xi \eta=M \xi \eta$. Thus $D_{L} \xi \dot{\zeta}=\dot{D}_{L} \xi M \xi \eta$. Hence Case 1-(IIa) holds in the definition of $m D_{L} \xi \eta$, and once again we get (ii).

This proves that (i) implies (ii), and the implication in the opposite direction is proved analogously; we shall omit the details. 
LemMA 2.8. Let $\sigma=y$ be any one of the four equations (Ia)-(IIb). Then for all $\xi, \zeta \in T e_{I \cup T}$ we have

$$
m(\sigma(\xi, \zeta))=m \zeta
$$

Proof. Because of 2.3 and 2.4 we can assume that $m \zeta=\zeta, m \xi=\xi$. Suppose $\sigma=y$ is (Ia) so that $\sigma(\xi, \zeta)=M \xi D_{L} \xi \zeta$. Let $m D_{L} \xi \zeta=\eta$. Then using 2.4 and 2.7(i), (ii) we get

$$
m(\sigma(\xi, \zeta))=m \boldsymbol{M} \xi m D_{L} \xi \zeta=m \boldsymbol{M} \xi \eta=\zeta=m \zeta
$$

as desired. The proofs for the other three equations are similar.

LEMMA 2.9. The following two conditions are equivalent for all $\tau, \tau^{\prime} \in \dot{T} \boldsymbol{T e}_{\mathrm{I} \cup \mathrm{T}}$ :

(i) $\tau \equiv_{\Phi} \tau^{\prime}$ where $\Phi=\Theta \mathscr{C}_{\rho} Q G$;

(ii) $m \tau=m \tau^{\prime}$.

Proof. It is a simple matter to prove by induction on the length of $\tau$ that $m \tau \equiv{ }_{\Phi} \tau$ for all $\tau \in T e_{r \cup r}$. This shows that (ii) implies (i).

To prove the implication in the opposite direction it suffices in view of the characterization of the relation $\equiv_{\Phi}$ described in the Preliminaries to prove that $\tau \hat{=}{ }_{\Gamma} \tau^{\prime}$ implies $m \tau=m \tau^{\prime}$ where $\Gamma$ is the set consisting of the equations (Ia)-(IIb), all of the equations of $\Theta$, and the definitions $Q v_{0} \cdots v_{x-1}=\rho Q$ for all $Q \in I$. Suppose that $\tau \hat{\curvearrowright}_{\Gamma} \tau^{\prime}$. Then there exist a $\pi \in T e_{I \cup T}$, a $v \in V a$, an assignment $\phi \in^{V a} T e_{I \cup T}$, and an equation $\sigma=\sigma^{\prime}$ such that either $\left(\sigma=\sigma^{\prime}\right) \in \Gamma$ or $\left(\sigma^{\prime}=\sigma\right) \in \Gamma$, and

$$
\tau=s u_{\xi}^{v} \pi \text { and } \tau^{\prime}=s u_{\xi^{\prime}}^{v} \pi \text { where } \xi=s u_{\phi} \sigma \text { and } \xi^{\prime}=s u_{\phi} \sigma^{\prime} .
$$

Thus, by Lemmas $2.4-2.6,2.8$, and the fact that $f$ is a normal-form function for $\Theta^{+}$, we have

$$
m \tau=m s u_{m \xi}^{v} \pi=m s u_{m \xi}^{v} \pi=m \tau^{\prime}
$$

This proves the lemma.

Lemmas 2.3 and 2.9 together show that $\boldsymbol{m}$ is a normal-form function for $\Theta \mathscr{C}_{\rho} Q G$. From 2.6 we get that $m$ coincides with $f$ on $T e_{I}$. Hence 2.1 (ii) holds. Finally $y_{\zeta}$ to see that 2.1 (iii) also holds, consider any $\tau, \tau^{\prime} \in T e_{I}$ and $\sigma_{0}, \cdots, \sigma_{\kappa-1} \in$ $T e_{I \cup T}$ satisfying the hypothesis of 2.1(iii). Clearly $m \tau=m \tau^{\prime}$ implies $m\left(\tau\left(\sigma_{0}, \cdots, \sigma_{\kappa-1}\right)\right)=m\left(\tau^{\prime}\left(\sigma_{0}, \cdots, \sigma_{\kappa-1}\right)\right)$. To prove the implication in the opposite direction let $\pi, \pi^{\prime} \in T e_{I}^{+}$be obtained from $\tau, \tau^{\prime}$, respectively, by simultaneously replacing all occurrences of $v_{0} ; \cdots, v_{\kappa-1}$ by $\chi^{-1} \sigma_{0}, \cdots, \chi^{-1} \sigma_{\kappa-1}$, respectively. Then we have

$$
\tau\left(\sigma_{0}, \cdots, \sigma_{\kappa-1}\right)=s u_{x} \pi, \tau^{\prime}\left(\sigma_{0}, \cdots, \sigma_{\kappa-1}\right)=s u_{x} \pi^{\prime}
$$


Since $\sigma_{0}, \cdots, \sigma_{\kappa-1}$ are distinct by hypothesis, the variables $\boldsymbol{X}^{-1} \sigma_{0}, \cdots, \chi^{-1} \sigma_{\kappa-1}$ are distinct from one another. Thus

$$
m \pi=m \pi^{\prime} \text { implies } m \tau=m \tau^{\prime} .
$$

Furthermore, by hypothesis the $\sigma_{0}, \cdots, \sigma_{\kappa-1}$ are either variables or have their principal operation symbols in $T$, and $m \sigma_{\lambda}=\sigma_{\lambda}$ for all $\lambda<\kappa$. This immediately gives $\pi, \pi^{\prime} \in T e_{I}^{++}$. Hence, by the definition of $\bar{f}$,

$$
\bar{f} s u_{x} \pi=s u_{x} f \pi ; \bar{f} s u_{x} \pi^{\prime}=s u_{x} f \pi^{\prime} .
$$

When combined with Lemma 2.6, conditions (15) and (17) show that the equality $\boldsymbol{m \tau}\left(\dot{\sigma}_{0}, \cdots, \sigma_{\kappa-1}\right)=m \tau^{\prime}\left(\sigma_{0}, \cdots, \sigma_{\kappa-1}\right)$ implies $s u_{x} f \pi=s u_{x} f \pi^{\prime}$. But, since $f$ is variable-minimizing, $f \pi, f \pi^{\prime} \in T e_{I}^{++}$because $\pi, \pi^{\prime} \in T e_{I}^{++}$. Thus from (1) and 2.6 we conclude that

$$
m \pi=f \pi=f \pi^{\prime}=m \pi^{\prime} ;
$$

hence $m \tau=m \tau^{\prime}$ by (16). Therefore, 2.1(iii) holds and the proof of Theorem 2.1 is complete.

\section{Applications}

In this section we apply Theorem 2.1 to obtain the universal embedding results for quasigroups discussed in the Introduction. We then state without proof a number of consequences of this universal property of quasigroups that are established in $\mathscr{U} \mathscr{E} \mathscr{T}$ for universal varieties in general. We also outline the proof of a certain improved version of 2.1 and discuss briefly its consequences. The section closes with some remarks on the possibility of extending the results of this paper to certain subvarieties of quasigroups.

For any variety $\mathscr{K}$ and any positive cardinal $\alpha$ we take $\mathfrak{F r}_{\alpha} \mathscr{K}$ to be the free algebra of $\mathscr{K}$ with $\alpha$ generators. In our first theorem we make use of the fact the larger of any two cardinals $\alpha$ and $\beta$ coincides with their union $\alpha \cup \beta$. The first infinite cardinal is denoted by $\omega$.

THEOREM 3.1. Let I be any countable type and assume that $\rho$ is a special non-overlapping definition of $I$ in $T$. Then for every variety $\mathscr{K}$ of type I there exists a variety $\mathscr{L}$ of quasigroups such that

$$
\mathfrak{I r}_{p} \mathfrak{F} \mathfrak{r}_{\alpha} \mathscr{L} \cong \mathfrak{F r}_{\alpha \cup \infty} \mathscr{K}
$$

for every positive cardinal $\alpha$. Moreover, if I is finite, then $\Theta \mathscr{L}$ is recursive in $\Theta \mathscr{K}$.

Proof. Let $\Theta$ be the theory of $\mathscr{K}$. Clearly we can assume that $\Theta$ is consistent. We also assume for simplicity that $\alpha \leqq \omega$ and thus that $\alpha \cup \omega=\omega$; the proof for an arbitrary cardinal is analogous. Let

$$
\Phi=\left(\Theta \mathscr{C}_{\rho} Q G\right) \cap E q_{\boldsymbol{T}}
$$


and take $\mathscr{L}$ to be the variety of all models of $\Phi$. Clearly $Q G \subseteq \Phi$ so $\mathscr{L}$ is a variety of quasigroups. Notice also that by 2.1(iii) we have

$\Phi$ is recursive in $\Theta$

if $I$ is finite.

Let $\mathcal{M}$ be the variety of all models of $\Theta \mathscr{C}_{\rho} Q G$. The theory $\Theta \mathscr{C}_{\rho} Q \boldsymbol{Q G}$ is the extension of $\Phi$ obtained by adjoining the definition $Q v_{0} \cdots v_{\kappa-1}=\rho Q$ for each $Q \in I$. Thus any algebra $\mathfrak{A} \in \mathscr{L}$ can be made into an algebra in $\mathcal{M}$ by adjoining, as the fundamental operations corresponding to the operation symbols $Q$ of $I$, the polynomials over $\mathfrak{A}$ defined by the terms $\rho Q$; furthermore, every element of $\mathcal{M}$ can be obtained in this way. It is now easy to see that



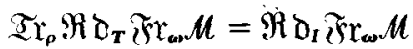

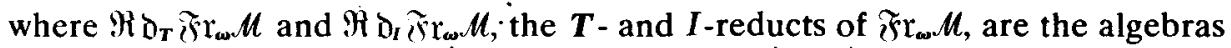
of type $T$ and $I$ respectively that are obtained from $\xi_{0}, \mathcal{M}$ by disregarding all fundamental operations except those corresponding to symbols in $T$ and $I$.

It is well known how to construct the free algebras of a variety by means of a normal-form function for the theory of the variety; indeed, free groups and rings are usually constructed in this way. Thus we can use the normal-form function $m$ given in 2.1 to construct $\tilde{F r}_{\omega} \mu$. Let

$$
\mathfrak{A}=\mathfrak{R} \mathfrak{D}_{\mathbf{I}} \mathfrak{F r} \boldsymbol{M} \text {. }
$$

The elements of $\mathscr{A}$, which are also the elements of $\mathfrak{F r}_{\omega} \mathcal{M}$, are taken to be the $m$-closed terms of type $I \cup T$, that is,

$$
A=\left\{\tau: \tau \in T e_{r \cup r}, m \tau=\tau\right\} .
$$

For every $Q \in I$ and all $\xi_{0}, \cdots, \xi_{\kappa-1} \in A$ we have $Q^{(\text {(x) }}\left(\xi_{0}, \cdots, \xi_{\kappa-1}\right)=$ $m Q \xi_{0} \cdots \xi_{\kappa-1}$. Let $X$ be the set of all $m$-closed terms in $T e_{r \cup T}$ which are either variables or have their principal operation symbol in $I$. Clearly $X$ generates $\mathfrak{A}$ and condition 2.1(iii) says that a relation can hold in $\mathfrak{A}$ between elements of $X$ just in case it holds identically in every member of $\mathscr{K}$. Therefore, since $X$ is of cardinality $\omega$, we have that $\mathfrak{A}$ is isomorphic to $\mathfrak{F r}_{\omega} \mathscr{K}$. The conclusion of the theorem now follows directly from (1)-(4).

THEOREM 3.2. The variety of quasigroups is effectively universal.

Proof. Let $I$ be any countable type and let $\mathfrak{A}$ be an algebra of type $I$. Assume first of all that $I$ is finite and $\mathfrak{A}$ is a free algebra over some variety $\mathscr{K}$, say

$$
\mathfrak{A} \cong \mathfrak{F} \mathfrak{r}_{\alpha} \mathscr{K} \text {. }
$$

We assume without loss of generality that 
Let $\rho$ be any special non-overlapping definition of $I$ in $\{M\}$; such a $\rho$ exists by 1.4. Then by 3.1 there exists a variety $\mathscr{L}$ of quasigroups such that $\mathfrak{D r}_{p} \mathfrak{F} \mathfrak{r}_{\alpha} \mathscr{L} \cong$ $\mathfrak{f r}_{\alpha} \cup \omega \mathscr{K}$ and $\Theta \mathscr{L}$ is recursive in $\Theta \mathscr{K}$. Take

$$
\mathfrak{B}=\mathfrak{乛} \mathrm{r}_{\alpha} \cup \operatorname{ar} \mathscr{L} \text {. }
$$

Then it follows immediately from (5) and (6) that all three of the conditions of 1.1 hold (with $\Theta \mathscr{K}$ replaced by $\boldsymbol{Q G}$ in 1.1(iii)).

We now drop the assumption that $I$ is finite and $\mathfrak{A}$ is free. To complete the proof we must find a quasigroup $\mathfrak{B}$ satisfying conditions 1.1 (i), (ii).

Let $J$ be a type disjoint from $I$ and consisting exclusively of unary operation symbols, in fact, one such symbol $P_{a}$ for each $a \in A$. Let $\Delta \subseteq E q$, be the set of all the equations

$$
Q P_{a_{0}} x \cdots P_{a_{k-1}} x=P_{b} x
$$

where $Q \in I, a_{0}, \cdots, a_{\kappa-1}, b \in A$, and $Q^{(x)}\left(a_{0}, \cdots, a_{\kappa-1}\right)=b$. For each finite $\Gamma \subseteq \Delta$ let $J_{\Gamma}$ be the set of all $P_{a}$ which occur in at least one member of $\Gamma$, and let $\mathscr{K}_{\Gamma}$ be the variety of type $I \cup J_{\Gamma}$ consisting of all models of

$$
\boldsymbol{\Theta A} \cup \Gamma \cup\left\{\boldsymbol{P}_{a} \boldsymbol{x}=\boldsymbol{P}_{a} \boldsymbol{y}: \boldsymbol{P}_{a} \in J_{\Gamma}\right\} .
$$

Clearly we have

$$
\boldsymbol{\Theta} \mathscr{K}_{\Gamma} \cap E q_{I}=\boldsymbol{\Theta} \mathscr{U} .
$$

Since $I \cup J_{\Gamma}$ is countable, there exists for each finite $\Gamma \subseteq \Delta$ a special non-overlapping definition $\pi_{\Gamma}$ of $I \cup J_{\Gamma}$ in $\{\boldsymbol{M}\}$. Furthermore, it is clear that these definitions can be chosen so that they all coincide on $I$; let $\rho$ denote this common restriction to $I$. By 3.1 there exists for each finite $\Gamma \subseteq \Delta$ a variety $\mathscr{L}_{\Gamma}$ of quasigroups such that

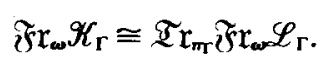

Observe that from (7) and (8) we get

$$
\boldsymbol{\Theta} \mathfrak{A}=\boldsymbol{\Theta} \mathfrak{T r}_{p} \mathfrak{F r} \mathfrak{r}_{\omega} \mathscr{L}_{\Gamma} \text { for each finite } \Gamma \subseteq \Delta .
$$

Let $\mathscr{L}$ be the least upper bound, in the lattice of quasigroup varieties, of the $\mathscr{L}_{\Gamma}$ for all finite $\Gamma \subseteq \Delta$. It follows from (9) that

$$
\boldsymbol{\Theta} \mathfrak{U}=\boldsymbol{\Theta} \mathfrak{T}_{\boldsymbol{p}} \mathfrak{F} \mathrm{r}_{\text {as }} \mathscr{L} \text {. }
$$

It is clear that every finite partial subalgebra of $\mathfrak{A}$ can be isomorphically embedded in $\mathfrak{R}_{D_{I}} \mathfrak{F}_{\omega_{\omega}} \mathscr{K}_{\Gamma}$ for a suitably chosen finite $\Gamma \subseteq \Delta$. Thus, by (8), each such finite partial subalgebra is isomorphically embeddable in the $\rho$-transform of a model of $\mathscr{L}$. Applying the compactness theorem of first-order predicate logic 
we conclude finally that there exists a $\mathfrak{B} \in \mathscr{L}$ satisfying condition $1.1(\mathrm{i})$; in view of (10) it follows automatically that 1.1(ii) is also satisfied. This completes the proof of the theorem.

A result somewhat stronger than 3.2 can be obtained. Consider the new notion of an effective universal variety that is obtained from 1.1 by deleting the condition "whenever $\mathfrak{A}$ is a free algebra over some variety" in the statement of 1.1(iii). As indicated in the Introduction, the variety of quasigroups is effectively universal in this stronger sense. This result however cannot be obtained directly from 3.1, or even 2.1. The reason for this stands out rather clearly in the proof of 3.2. In order to obtain an isomorphic embedding of an arbitrary algebra $\mathfrak{A}$ in a definitional transform of some quasigroup by means of 3.1 alone, we are required to interpret the elements of $\mathfrak{U}$ as (constant unary) polynomials in some relatively free quasigroup $\mathfrak{B}$. Consequently, the theory of identities of $\mathfrak{B}$ must necessarily include an interpretation of the word problem for $\mathfrak{A}$. Thus, in general, the theory of identities of $\mathfrak{B}$ cannot be recursive in the corresponding theory of $\mathfrak{A}$ since, for example, it is easy to construct an $\mathfrak{A}$ such that the latter theory is recursive while the word problem for $\mathfrak{A}$ in unsolvable.

The difficulty can be overcome by extending our basic lemma 2.1 . It is useful to think of this extension as a kind of parametrized version of 2.1. Although the version we now describe is not the most general one available, it suffices for the purpose at hand. Compare Theorem 4.20 of $\mathscr{U} \mathscr{E T}$.

Let $I, \Theta$, and $\rho$ be as in the hypothesis of 2.1. Let $\mathfrak{A}$ be any model of $\Theta$. For each $a \in A$ let $P_{a}$ be a new unary operation symbol and let $J$ and $\Delta$ be defined as in the proof of 3.2. Let

$$
\begin{aligned}
\Theta_{\mathfrak{x}} & =\Theta_{I \cup J}\left[\Theta \cup \Delta \cup\left\{P_{a} x=P_{a} y: a \in A\right\}\right], \\
Q G_{\dot{q}} & =\Theta_{T \cup \mathcal{J}}\left[Q G \cup\left\{P_{a} x=P_{a} y: a \in A\right\}\right] .
\end{aligned}
$$

Finally let $\rho_{\mathrm{a}}$ be the extension of the definition $\rho$ to $I \cup J$ that is obtained by setting $\rho P_{a}=P_{a} x$ for each $a \in A$. Then we can conclude that there exists a transformation $m$ of $T e_{r \cup r \cup J}$ satisfying conditions 2.1 (i)-(iv) with $I, \Theta, \rho$, and $Q G$ replaced respectively by $I \cup J, \Theta_{\mathfrak{x}}, \rho_{\mathfrak{x}}$, and $\boldsymbol{Q} G_{\mathfrak{r}}$. In addition, the following condition is satisfied provided $I$ is finite.

\section{(v) $m$ restricted to $T e_{I \cup T}$ is recursive in $\Theta$.}

The proof of this result is a straightforward modification of that of 2.1.

In $\mathscr{U} \mathscr{E} \mathscr{T}$ a number of results about universal and effectively universal varieties are obtained. We shall state several of them here for quasigroups without proof and refer the reader to the corresponding theorems in $\mathscr{U E T}$.

THEOREM 3.3. There exist a continuum number of universal varieties of 
quasigroups any two of which have only trivial one-element quasigroups in common.

See Theorem 3.9 of $\mathscr{U} \mathscr{E} \mathscr{T}$.

THEOREM 3.4. For each recursively enumerable degree of unsolvability $\delta$ there exists a variety $\mathscr{K}$ of quasigroups defined by a single equation such that $\Theta \mathscr{K}$ is of degree $\delta$. Furthermore, $\mathscr{K}$ can be taken so that $\delta$ is also the degree of the set of all $\varepsilon \in \Theta \mathscr{K}$ such that $|V a \varepsilon|=1 .^{2}$

For arbitrary effectively universal varieties this result is established in Theorem 3.10 of $\mathscr{U} \mathscr{E} \mathscr{T}$. The following interesting consequence of this result was pointed out by Boone and Rogers (1966); see $\mathscr{U} \mathscr{E} \mathscr{T}$, Theorem 3.11.

THEOREM 3.5. Let $\Gamma$ be the set of all $\varepsilon \in E q_{\mathrm{r}}$ such that

(i) every model of $\varepsilon$ is a quasigroup, and

(ii) $\Theta_{T}[\varepsilon]$ is decidable.

Then $\Gamma$ is a maximal $\Sigma_{3^{-}}$set in the Kleene-Mostowski hierarchy. In particular, $\Gamma$ fails to be recursively enumerable. ${ }^{2}$

Some of the results obtained in $\mathscr{U} \mathscr{E} \mathscr{T}$ are established only for a special class of universal varieties - the so-called normal universat, or $\boldsymbol{n}$-universal, varieties. Most of these results, however, are essentially consequences only of the following property of an arbitrary $n$-universal variety $\mathscr{L}$ : for each countable type $I$ there exists a definition $\rho$ of $I$ in the type $J$ of $\mathscr{L}$ such that the conclusion of Theorem 2.1 holds with $Q G$ and $T$ replaced by $\Theta \mathscr{L}$ and $J$, respectively. In particular, the proofs of these results given in $\mathscr{U} \mathscr{E} \mathscr{T}$ can with little difficulty be modified so as to apply to the variety of quasigroups. Some results of this kind are formulated in the next two theorems. The first one, Theorem 3.6, further describes the relationship between the structures of the free algebras $\mathfrak{F r}_{\alpha} \mathscr{L}$ and $\mathfrak{F r}_{\beta} \mathscr{K}$ discussed in Theorem 3.1. Cf. Theorem 5.2 of $\mathscr{U} \mathscr{E} \mathscr{T}$.

Let $I, \rho$, and $\mathscr{K}$ be as in the statement of 3.1. It is easy to see that the particular variety $\mathscr{L}$ constructed in the proof of 3.1 is the largest one possible satisfying the conclusion of the theorem in the sense that it includes every variety with this property as a subvariety. We denote this particular variety $\mathscr{L}$ by $\hat{\rho} \mathscr{K}$. It follows easily from 3.1 together with its proof that, for any positive cardinal $\alpha$, any one-one mapping from the free generators of $\mathfrak{F r}_{\alpha} \mathscr{K}$ onto the free generators of $\mathfrak{F r}_{\alpha}(\hat{\rho} \mathscr{K})$ can be extended to an isomorphism from $\mathfrak{F r}_{\alpha} \mathscr{K}$ into $\mathfrak{I x}_{\rho} \mathfrak{F} \mathfrak{r}_{\alpha}(\hat{\rho} \mathscr{K})$. In the following theorem we shall identify $\mathfrak{i} \mathfrak{r}_{\alpha} \mathscr{K}$ with its isomorphic image in $\mathfrak{I}_{\mathfrak{r}_{\boldsymbol{\rho}}} \mathfrak{F} \mathfrak{r}_{\alpha}(\hat{\rho} \mathscr{K})$.

THEOREM 3.6. Let I be any countable type and assume $\rho$ is a special non-overlapping definition of $I$ in $T$. Let $\mathscr{K}$ be any variety of type $I$ and $\alpha$ any positive cardinal. Then for each subalgebra $\mathfrak{A}$ of $\mathfrak{F r}_{\alpha} \mathscr{K}$ there exists a subquasigroup $\mathfrak{B}$ of the relatively free quasigroup $\mathcal{F} r_{\alpha}(\hat{\rho} \mathscr{K})$ such that $B \cap F r_{\alpha} \mathscr{K}=A$. 
This theorem plays a principal role in the proof that there does not exist a recursive algorithm for deciding whether or not a given finite set of equations defines, together with the equations (Ia)-(IIb), a Schreier variety of quasigroups. This result is established in Pigozzi (b). The analogous result for the amalgamation property is also established there; it depends on the next theorem which corresponds to Theorem 5.3 of $U \mathscr{E} \mathscr{T}$.

Let $\mathscr{K}$ be an arbitrary variety and let $\rho$ be a special non-overlapping definition of the type $I$ of $\mathscr{K}$ in $\boldsymbol{T}$. It is easily seen that the $\rho$-transform $\mathfrak{I} r_{\rho}$ becomes the object map of a functor from $\hat{\rho} \mathscr{K}$ into $\mathscr{K}$ when these two varieties are considered as categories in the natural way. It is well known that the functor $\mathfrak{I}_{\mathfrak{p}}$ possesses a left-adjoint. A construction of this left-adjoint is given in $\mathscr{U} \mathscr{E} \mathscr{T}$ where it is denoted by $\mathfrak{H}_{\rho}$, the universal inverse $\rho$-transform.

TheORem 3.7. Assume $I, \rho$, and $\mathscr{K}$ are as in Theorem 3.6, and assume $\mathfrak{A}, \mathfrak{B} \in \mathscr{K}$. If $\mathfrak{A}$ is a subalgebra of $\mathfrak{B}$, then $\mathfrak{U t}_{\mathfrak{i}} \mathfrak{A}$ is isomorphic to a subalgebra of $\mathfrak{H i}_{\mathfrak{p}} \mathfrak{B}$. More generally, if $h: \mathfrak{A} \rightarrow \mathfrak{B}$ is an isomorphism, then so is

$$
\bar{h}: \mathfrak{U i}_{\rho} \mathscr{H} \rightarrow \mathfrak{U i}_{\rho} \mathfrak{B}
$$

where $\bar{h}$ is the image of $h$ under the functor $\mathfrak{H i}_{\rho}$.

COROLlaRY 3.8. Let $I, \rho$, an $\dot{\alpha}$ be as in 3.6. If $\hat{\rho} \mathscr{K}$ has the amalgamation property, then so does $\mathscr{K}$.

Our final theorem is easily seen to be a generalization of Theorem 2.1, and we shall only outline its proof since it is an extension of the proof of 2.1.

THEOREM 3.9. Assume $\Gamma$ is any finite set of equations of type $\boldsymbol{T}$ that fail to be in QG. Then there exists a finitely based extension $\Phi$ of $Q G$ satisfying the following conditions.

(i) $\Phi$ and $\varepsilon$ are mutually inconsistent for each $\varepsilon \in \Gamma$.

(ii) Let I be any countable type. Then there exists a definition $\rho$ of $I$ in $T$ such that, for each theory $\Theta$ of type $I$, there exists a transformation $m$ of $T e_{I \cup T}$ satisfying conditions 2.1(i)-(iv) with QG replaced everywhere by $\Phi$.

Proof. We shall assume that $\Gamma$ consists of a single equation $\gamma_{0}=\gamma_{1}$; the argument in the general case is more complicated in details but presents no essentially new difficulties. We also assume without loss of generality that for $\mu=0,1$.

$$
\gamma_{\mu} \equiv \text { oG } \delta \text { implies }\left|\gamma_{\mu}\right| \leqq|\delta| \text { for all } \delta \in T e_{T} .
$$

Let $\lambda$ be the largest natural number $\mu$ such that the variable $v_{\mu}$ occurs in either $\gamma_{0}$ or $\gamma_{1}$. Let $S, P_{0}, \cdots, P_{\lambda}$ be operation symbols distinct from each other 
and from the symbols in $T$ and such that $S$ is of rank 4 and $P_{0}, \cdots, P_{\lambda}$ are all of rank 1. Let

$$
J=\left\{S, P_{0}, \cdots, P_{\lambda}\right\}
$$

and let $\pi$ be a special non-overlapping definition of $J$ in $\{\boldsymbol{M}\}$ having the following two properties:

$$
\gamma_{0}\left(\pi P_{0}(x), \cdots, \pi P_{\lambda}(x)\right) \neq \gamma_{1}\left(\pi P_{0}(x), \cdots, \pi P_{\lambda}(x)\right)
$$

for each countable type $I$ disjoint from $J$ there exists a definition $\rho$ of $I$ in $\{M\}$ such that $\rho \cup \pi$ is a special non-overlapping definition of $I \cup J$ in $\{\boldsymbol{M}\}$ and

$$
|(\rho \cup \pi) Q|>\left|\gamma_{0}\right|,\left|\gamma_{1}\right| \text { for every } Q \in I \cup J .
$$

That such a definition $\pi$ exists follows without difficulty from Lemma 1.4.

Let

$$
\delta_{0}=\gamma_{0}\left(\pi P_{0}(x), \cdots, \pi P_{\lambda}(x)\right), \delta_{1}=\gamma_{1}\left(\pi P_{0}(x), \cdots, \pi P_{\lambda}(x)\right),
$$

and let $\Phi$ be the theory of quasigroups generated by (Ia)-(IIb) together with the equations

$$
\begin{array}{r}
\pi P_{\mu}(x)=\pi P_{\mu}(y) \text { for } \mu=0, \cdots, \lambda, \\
\pi S(x, x, y, z)=y, \text { and } \pi S\left(\delta_{0}, \delta_{1}, y, z\right)=z .
\end{array}
$$

It is clear that $\Phi$ and $\delta_{0}=\delta_{1}$ are mutually inconsistent, so (i) holds. Let $I$ be any countable type disjoint from $T$; we assume without loss of generality that $I$ is also disjoint from $J$. Let $\rho$ be any special non-overlapping definition of $I$ in $\{\boldsymbol{M}\}$ such that $\rho \cup \pi$ is a special non-overlapping definition of $I \cup J$ in $\{\boldsymbol{M}\}$ satisfying (13). Finally, let $\Theta$ be any consistent theory of type $I$. We shall construct a transformation $m$ satisfying the conclusion of (ii).

The construction of $m$ follows closely the construction given in the proof of 2.1. In particular, we begin by choosing a variable-minimizing normal-form function for the extended theory $\Theta^{+}$. Observe that by (11) we have:

(15) for all $\xi, \eta \in T e_{T}, \sigma(\xi ; \eta) \nless \gamma_{0}, \gamma_{1}$ for every $\sigma \in T e_{T}$ such that $\sigma=y$ is one of the equations (Ia)-(IIIb)

The transformation $m$ is constructed by recursion on the length of $\tau \in T e_{r \cup \tau} . m \tau=\tau$ if $\tau \in V a$. Assume. $|\tau|>1$. The definition now separates into fifteen cases. The first five cases are identical to Cases 1-5 of the proof of 2.1.

CASE 6: $m^{*} \tau=\pi P_{\mu}(\xi)$ for some $\mu \leqq \lambda$. Take $m \tau=\pi P_{\mu}(x)$.

CASE 7: $m^{*} \tau=D_{L}\left(\pi P_{\mu}\right)_{L}(\xi) \pi P_{\mu}(x)$ for $\mu \leqq \lambda$. Take $m \tau=\left(\pi P_{\mu}\right)_{R}(\xi)$.

CASE 8: $m^{*} \tau=D_{R} \pi P_{\mu}(x)\left(\pi P_{\mu}\right)_{R}(\xi)$ for $\mu \leqq \lambda$. Take $m \tau=\left(\pi P_{\mu}\right)_{L}(\xi)$. 
CASE 9: $m^{*} \tau=\pi S(\xi, \xi, \eta, \xi)$. Take $m \tau=\eta$.

CASE 10: $m^{*} \tau=D_{L} \pi S_{L}(\xi, \xi, \eta, \zeta) \eta$. Take $m \tau=\pi S_{R}(\xi, \xi, \eta, \zeta)$.

CASE 11: $m^{*} \tau=D_{R} \eta\left(\pi S_{R}(\xi, \xi, \eta, \zeta)\right)$. Take $m \tau=\pi S_{L}(\xi, \xi, \eta, \zeta)$.

CASE 12: $m^{*} \tau=\pi S\left(\delta_{0}, \delta_{1}, \eta, \zeta\right)$. Take $m \tau=\zeta$.

CASE 13: $m^{*} \tau=D_{L} \pi S_{L}\left(\delta_{0}, \delta_{1}, \eta, \zeta\right) \zeta$. Take $m \tau=\pi S_{R}\left(\delta_{0}, \delta_{1}, \eta, \zeta\right)$.

CASE 14: $m^{*} \tau=D_{R} \zeta\left(\pi S_{R}\left(\delta_{0}, \delta_{1}, \eta, \zeta\right)\right)$. Take $m \tau=\pi S_{L}\left(\delta_{0}, \delta_{1}, \eta, \zeta\right)$.

CASE 15: None of the first fourteen cases apply. Take $m \tau=m^{*} \tau$.

The demonstration that $m$ is well defined by these conditions follows closely the corresponding demonstration in the proof of 2.1. In particular it relies heavily on the fact that $\rho \cup \pi$ is a special non-overlapping definition. The only real novelty occurs in showing that Cases 9 and 12 (also Cases 10 and 13 and Cases 11 and 14) cannot simultaneously apply; this of course depends on the fact that $\delta_{0}, \delta_{1}$ are distinct terms by (12) and (14).

The proof that $m$ is a normal-form function for $\Theta \mathscr{C}_{\rho} \Phi$ is also very similar to the proof of the same result in 2.1 with $\Phi=\boldsymbol{Q G}$. Lemmas $2.2-2.8$ and their proofs transfer mutatis mutandis to the present case. The proof of 2.9 however requires that we show the following hold for all $\xi, \eta, \zeta \in T e_{\text {Iur }}$ :

$$
\begin{gathered}
m\left(\pi P_{\mu}(\xi)\right)=m\left(\pi P_{\mu}(\eta)\right) \text { for all } \mu \leqq \lambda, \\
m(\pi S(\xi, \xi, \eta, \zeta))=m \eta, \\
m\left(\pi S\left(\delta_{0}, \delta_{1}, \eta, \zeta\right)\right)=m \zeta .
\end{gathered}
$$

The proofs of (16) and (17) are straightforward, and the proof of (18) depends on the fact that $m \delta_{0}=\delta_{0}, m \delta_{1}=\delta_{1}$. But these equalities are easily proved using (13) and (15); in particular, observe that, because of (13) and the non-overlapping property of $\rho \cup \pi$, no substitution instance of any $(\rho \cup \pi) Q$ with $Q \in I \cup J$ can occur as a subterm of $\delta_{0}$ or $\delta_{1}$ unless it is one of the terms $\pi P_{\mu}(x)$ that were substituted for the variables in $\gamma_{0}, \gamma_{1}$ to form $\delta_{0}, \delta_{1}$.

Lemma 2.9 is now proved in the same way as before and this immediately gives 2.1(i) (with $\boldsymbol{Q} \boldsymbol{G}$ replaced by $\Phi$ ). Conditions 2.1 (ii)-(iv) now follow easily as in the proof of 2.1; we omit the details. This completes the proof of the theorem.

Theorem 3.1 can now be improved by requiring that every non-trivial quasigroup in the variety $\mathscr{L}$ fail to satisfy identically each member of any finite set of non-identities of quasigroups given in advance (additional restrictions must be put on the definition $\rho$ however). Analogous improvements of Theorems 3.2-3.8 are readily formulated. As an immediate corollary of the improved version of 3.2 we get the following theorem originally proved by Bol'bot (1972).

THEOREM 3.10. Let $L$ be any finite set of proper subvarieties of the variety of all quasigroups. Then there exists a continuum number of equationally complete 
quasigroup varieties $\mathscr{K}$ such that $\mathscr{K} \cap \mathscr{L}$ contains only one-element quasigroups for each $\mathscr{L} \in L$.

Theorems 3.9 and 3.10 also hold for all normal universal varieties, in particular, commutative groupoids and bisemigroups.

In our final remarks we would like to discuss the possibility of extending the results of this paper to important subvarieties of quasigroups. The most natural one to consider is that of loops; a loop is a quasigroup which satisfies the identity

$$
D_{L} x x=D_{R} y y .
$$

The variety of loops is not universal since, like all varieties each of whose members includes a one-element subalgebra, all constant unary polynomials over an arbitrary loop are identical. However, as should be expected, loops do inherit some universal-like properties from quasigroups. For example, we know that loops are universal for non-constant multi-unary algebras in the sense that Theorem 2.1 continuous to hold after the following changes are made in its statement: (I) $I$ is restricted so as to contain only operation symbols of rank 1 ; (II) $\Theta$ contains no equation of the form $\tau(x)=\sigma(y)$; (III) $Q G$ is replaced everywhere by the theory of loops. The proof of the modified version of 2.1 follows pretty closely the proof of the original.

This partial result allows some of the consequences of 2.1 given in Section 3 to be extended to loops; this applies in particular to Theorems 3.4 and 3.5. We have not systematically investigated the universal properties of loops, however, and we think this would be an interesting problem to consider.

The variety of commutative groupoids is known to be universal and we think that it is very probable that the same is true of commutative quasigroups. The result for commutative groupoids is obtained in $\mathscr{U E F}$, Theorem 4.25. The method of proof differs considerably from that of Theorem 3.2, but it seems likely that the two proofs can be combined to give the universality of commutative quasigroups.

A quasigroup is totally symmetric if its three fundamental operations of multiplication, left-division, and right-division all coincide. These quasigroups form an important subvariety of quasigroups but we have not investigated their universal properties and have no feeling at all as to how extensive they may be. Bol'bot (1967) has shown, however, that there exists a continuum number of equationally complete varieties of totally symmetric quasigroups.

The variety of groups fails to be universal in a very strong sense. For it appears that even the weakest kind of universal property implies the existence of a continuum number of complete subvarieties, but it is well known that there exist only countably many complete group varieties. As to be expected the associative law seems to be highly destructive of universal properties, and thus it 
would be particularly interesting to investigate the universal properties of a variety of quasigroups, such as Moufang loops, which satisfy a quasi-associative identity. We have not as yet done any work in this direction.

\section{References}

A. D. Bol'bot (1967), 'Equationally complete varieties of totally symmetric quasigroups', (In Russian.) Algebra i Logika 6, 13-19.

A. D. Bol'bot (1972), 'Varieties of quasigroups', (In Russian.) Sibirsk. Mat. Z. 13, 252-271. [English Translation. Siberian Math. J. 13 (1972), 173-186.]

W. W. Boone and H. Rogers Jr. (1966), 'On a problem of J. H. C. Whitehead and a problem of Alonzo Church', Math. Scand. 19, 185-192.

T. Evans (1951), 'On multiplicative systems defined by generators and relations. I. Normal form theorems', Proc. Cambridge Philos. Soc. 47, 637-649.

T. Evans (1971), 'Identical relations in loops, I', J. Austral. Math. Soc. 12, 275-286.

A. I. Mal'cev (1939), 'On the embedding of associative systems into groups. I', (In Russian.) Mat. Sb. 6, 331-336.

A. I. Mal'cev (1966), 'Identical relations in varieties of quasigroups', (In Russian.) Mat. Sb. 69, 3-12. [English translation. Amer. Math. Soc. Transl. (2), 82 (1969), 225-235. Also in: A. I. Mal'cev, The metamathematics of algebraic systems (North-Holland Publishing Co., Amsterdam, 1971).]

G. F. McNulty, 'The decision problem for equational bases of algebras', Ann. Math. Logic (to appear).

H. Neumann (1967), Varieties of groups (Ergebnisse Der Mathematik und Ihrer Grenzgebiete, Bd. 37, Springer-Verlag, Berlin).

P. M. Neumann and J. Wiegold (1964), 'Schreier varieties of groups', Math. Z. 85, 392-400.

D. Pigozzi (1973), 'On the decision problem for equational theories of quasi-groups', Notices Amer. Math. Soc. 20, A-462.

D. Pigozzi (a), 'Universal equational theories and varieties of algebras', (to appear).

D. Pigozzi (b), 'Base-undecidable properties of universal varieties', (to appear).

A. Tarski (1968), 'Equational logic and equational theories of algebras', in: H. A. Schmidt, K. Schutte, H.-J. Thiele, eds., Contributions to mathematical logic (North-Holland Publishing Co., Amsterdam).

\section{Department of Mathematics}

Iowa State University

Ames, Iowa 50010

U.S.A. 